\title{
Low-velocity impact damage characterization of carbon fiber reinforced polymer (CFRP) using infrared thermography
}

\author{
Yin Li, Wei Zhang ${ }^{*}$, Zheng-wei Yang, Jin-yu Zhang, Sheng-jie Tao
}

Xi'an Research Institute of High-Tech, Hongqing Town, Xi'an 710025, PR China

\begin{abstract}
ABSTEACT
Carbon fiber reinforced polymer (CFRP) after low-velocity impact is detected using infrared thermography, and different damages in the impacted composites are analyzed in the thermal maps.

The thermal conductivity under pulse stimulation, frictional heating and thermal conductivity under ultrasonic stimulation of CFRP containing low-velocity impact damage are simulated using numerical simulation method. Then, the specimens successively exposed to the low-velocity impact are respectively detected using the pulse infrared thermography and ultrasonic infrared thermography. Through the numerical simulation and experimental investigation, the results obtained show that the combination of the above two detection methods can greatly improve the capability for detecting and evaluating the impact damage in CFRP. Different damages correspond to different infrared thermal images. The delamination damage, matrix cracking and fiber breakage are characterized as the block-shape hot spot, line-shape hot spot, and "工" shape hot spot respectively.
\end{abstract}

Keywords:

Infrared thermography

Infrared thermal images

Carbon fiber reinforced polymer (CFRP)

Low-velocity impact damage characterization

\section{Introduction}

Composite materials have been proved as an important structural material for aerospace and other fields due to their excellent performances of high specific strength, high specific modulus, high temperature resistance, corrosion resistance and fatigue resistance, etc. [1]. However, the composite structure is extremely sensitive to the impact, as a result, even the imperceptible impact on the surface can lead to such internal damages as matrix cracking, fiber breakage and delamination, reducing the bearing capacity of the composite structures greatly $[2,3]$. So it is vital to explore the effective detection method.

Many techniques have been applied to detect the impact damage, among which the most common detection methods are based on ultrasonic inspection [4], radiographic inspection [5] and acoustic emission [6, 7]. But the above methods require abundant experienced technicians to analyze the information obtained. Furthermore, the radiographic inspection method does a lot of harms to the body.

Infrared thermography possesses superior performances such as high-speed, non-contact, large area inspection and intuitional results etc. in detecting the hackneyed-defects of composite materials and attracts extensive attention in the world [8-12]. In 1984, Milne and Reynolds [13] proposed the infrared thermography detection method and pointed out its ability to inspect defects in the materials with great speed and without physical contact. As a consequence, many scholars started to investigate this nondestructive testing (NDE) technique theoretically. Tamma and Yurko [14] utilized three-dimensional units with different features to analyze the thermal conductivity on the unidirectional composite materials, but the model built was imprecise and cost much time due to the limitation of the calculation method. To solve this problem, Savoia [15] employed analytical method to analyze the thermal conductivity for the composite laminates. But this method was difficultly applied to engineering because of the limitation of the boundary condition. Involving the linear thermal lamination theoretic model, Noack [16], supposing linear distribution for temperature along the thickness direction of the laminates, utilized the two-dimensional units to analyze thermal conductivity of three units, and on this basis, developed high order thermal lamination theory. Subsequently, infrared thermography was proven successful to detect and characterize impact damage of composite materials. Avdelidis [17] applied pulse infrared thermography to carbon fiber reinforced composites plates, honeycomb and sandwich structure, as a result, the defects

\footnotetext{
${ }^{*}$ Corresponding author. Address: Xi' an Research Institute of High-Tech, Hongqing Town, Xi'an 710025, PR China E-mail address: DIYLLLLY@163.com(Y. Li), zhangweihuaiyu@163.com(W.Zhang).
} 
nearby the surface of the specimen inspected could be effectively detected by the means of pulse infrared thermography. To evaluate the detection ability of infrared thermography, Ravikiran et al. [18] investigated the comparison on the impact damage detection in carbon fiber reinforced polymer (CFRP) between the ultrasound C-scan and infrared thermography. As a result, the ultrasound C-scan was superior to the infrared thermography in quantifying the impact damage of composite materials. Introducing infrared image processing, Usamentiaga [19] proposed a method to automatically detect impact damage in carbon fiber composites using infrared thermography. This method can correctly detect all the impact defects caused by energy from $6 \mathrm{~J}$ to $50 \mathrm{~J}$. But, the previous papers available did not describe the details of the low-velocity impact damage in CFRP by the infrared thermography and not report the matters concerning the combination of the pulse infrared thermography and ultrasonic infrared thermography applied to detect the defects in the materials.

Due to the characteristics of anisotropic and inhomogeneous of composite materials, the transmissible process of the ultrasonic, the heat generation and conduction patterns in the composite materials are more complicated than those in the metal. Therefore, it is necessary for the further investigation of low-velocity impact damage detection and characterization of composite materials using infrared thermography. In this work, the combination of pulse infrared thermography and ultrasonic infrared thermography is introduced as the nondestructive detection for the low-velocity impact damage of composite materials. The detection principle of the pulsed infrared thermography and ultrasonic infrared thermography is analyzed and the distribution law of surface temperature field after excitation is deducted. The low-velocity impact damage detection testing results of composite materials using the two thermal wave methods are analyzed based on the finite element analysis model containing the low-velocity impact damage of composite materials. Pulse infrared thermography and ultrasonic infrared thermography are applied to detect the low-velocity impact damage of the specimens made of carbon fiber composite materials. The attention of this work is paid to explore what low-velocity impact damage types the pulse infrared thermography and ultrasonic infrared thermography are respectively suitable for detecting and characterize the delamination, matrix cracking and fiber fracture in the impacted composites.

This work can provide references to identify the impact damage type in composite materials using infrared thermography and lay foundations for the quantitative detection of impact damage and maintenance of the composite materials.

\section{The detection principle of infrared thermography}

Infrared thermography is an active detection technology with the controllable source of thermal excitation such as pulsed, cycled and ultrasonic et al
[20-22]. It is applied to the detected objects, if there were defects in the specimen, the changeable temperature of the surface of the specimen will be generated and recorded by the infrared camera (IR). Rapid detection and quantitative identification of the defect inside the objects can be realized after the infrared image sequences collection, analysis and process [23]. Its detection principle can be showed in Fig. 1.

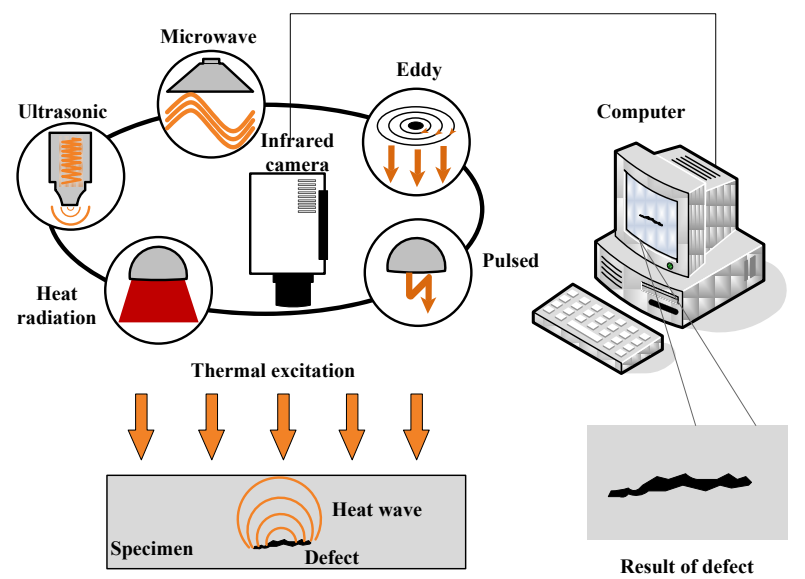

Fig. 1 Schematic diagram of thermal wave detection technology

According to the detection principle presented previously, the substance of detecting the defects using infrared thermography is to solve the heat conduction equation.

In allusion to the materials with the characteristics of homogeneous opaque and isotropy, the heat conduction equation is as follows [6].

$\alpha \frac{\partial^{2} T(x, t)}{\partial x^{2}}=\frac{\partial T(x, t)}{\partial t}$

With $\alpha=k /(\rho c)$, where $\alpha$ is the thermal diffusion efficiency, $k$ is the thermal-conductivity coefficient, $\rho$ is the density of specimen, and $c$ is the specific heat.

\subsection{Pulse infrared thermography}

In this work, a heat Dirac pulse represented by $q$ as shown in Eq. (2) is applied by a flashlight on the surface of isotropic infinite flat plate with a defect depth of $d$.

$$
q=q_{0} \delta(x) \delta(t)
$$

The following conditions at the border:

$-\left.k \frac{\partial T(x, t)}{\partial x}\right|_{x=0}=\left.q\right|_{x=0}=q_{0} \delta(t)$

The thermal exchanges containing convective exchange and radiant exchange are ignored.

$\left.\frac{\partial T(x, t)}{\partial x}\right|_{x=d}=0$ 
While, the initial condition is in Eq. (5)

$$
T(x, t)=T_{0}
$$

In the above equations, $T$ is the temperature on the surface of the specimen, $q$ is the heat Dirac pulse, $d$ is the depth of the plate.

The temperature in the defect area and non-defect region changes over time, and the temperature difference can be expressed by solving the Eqs. (1) - (5) based on the Laplace transform:

$\Delta T=\frac{q}{\sqrt{\pi \rho c k t}} e^{\frac{-d^{2}}{\alpha t}}$

By solving the extremum of Eq. (6), the maximum temperature difference, as shown in Eq. (7), is obtained at the optimal detecting time $t_{m}=2 d^{2} / \alpha$

$$
\Delta T_{m}=\frac{q}{\rho c \sqrt{2 \pi e} \cdot d}
$$

As is known from the above presentation, the defects in the specimen can cause the changeable temperature on the surface of the specimen under the action of the thermal excitation. The degree and changing process of the differences are closely related with the damage descriptive parameters. The defects can be identified by the temperature variations.

\subsection{Ultrasonic infrared thermography}

In the process of nondestructive detection by ultrasonic infrared thermography, the short ultrasonic pulse with low frequency is applied to specimen inspected. The ultrasonic wave spreads in the specimen after the interface coupling. Due to the friction in the defect or damage, the mechanical energy of the ultrasonic would be transformed into heat, leading to the rising temperature at the damage area and the area around the positions, which can be observed and recorded by IR [13].

The interfaces of interfacial joint damage in the structure are collided during the ultrasonic stimulation, where can be regarded as the multiple-degree freedom vibration system. And the ultrasonic mechanical energy is transformed into heat owing to the friction between the interfaces. The control equation of forced vibration under ultrasonic stimulation can be described as follow [24]:

$M \frac{d^{2} U}{d t^{2}}+C \frac{d U}{d t}+K U=F+R$

Where $U$ is the matrix of node displacement, $M$ is the matrix of quality, $C$ is the matrix of damping with the unit $\mathrm{kg} / \mathrm{s}, \mathrm{K}$ is the matrix of stiffness, $F$ is the external load matrix caused by the ultrasonic wave, $R$ is the contact force matrix of the damage interface. The contact interface is considered as the boundary. The boundary conditions are unknown and changeable with the contact deformation. Therefore, contact force of damage interface is a function of displacement. $R$ can be divided to the normal contact force and tangential friction force.

On the basis of Newton third law and thermodynamics first law, the heat flux density at the damage is as shown in Eq. (9):

$$
q(t)=\left[\mu_{d}+\left(\mu_{s}-\mu_{d}\right) e^{-c|v|}\right] F_{n}(t) v_{\tau}(t)
$$

Where $q(t)$ is the heat flux density, $\mu_{\mathrm{s}}$ and $\mu_{\mathrm{d}}$ are the static friction coefficient and dynamic friction coefficient respectively, $c$ is the velocity coefficient for the static friction turning into dynamic friction, $F_{N}(t)$ is the normal contact force, $v(t)$ is the tangential relative velocity of the contact point.

However, the temperature field of the composite material is three-dimensional due to its characteristics of anisotropic and inhomogeneous and the heat conduction equation is as follows.

$\frac{\partial T(x, y, z, t)}{\partial T(t)}=\alpha_{x} \frac{\partial T^{2}(x, y, z, t)}{\partial T^{2}(x)}+\alpha_{y} \frac{\partial T^{2}(x, y, z, t)}{\partial T^{2}(y)}+\alpha_{z} \frac{\partial T^{2}(x, y, z, t)}{\partial T^{2}(z)}$

It is difficult to solve the analytic solutions of the three-dimensional temperature fields. Therefore, the numerical simulation is applied to compute the three-dimensional temperature fields in the following the section.

\section{Numerical simulation}

The damage in the composite material caused by low-velocity impact mainly contains the matrix cracking, delamination and fiber breakage $[25,26]$. So the model of composite material containing the three damage types prefabricated has been built to numerically compute the three-dimensional temperature fields using pulse infrared thermography and ultrasonic infrared thermography.

3.1. Numerical simulation using pulse infrared thermography

The model of the carbon fiber reinforced plate (CFRP) containing the matrix cracking, delamination and fiber breakage has been built. The composite materials are stacked in twelve layers with the size of $150 \mathrm{~mm} \times 100 \mathrm{~mm} \times 4 \mathrm{~mm}$ and extended in $\left[0^{\circ} / 45^{\circ} / 90^{\circ} /-45^{\circ}\right]_{12}$. For convenience to the research, the following assumptions are supposed in modeling:

(1) The bonding situations between the unidirectional plates are perfect and the influence of thermal resistance is not taken into consideration.

(2) Energy conservation and temperature continuity should be satisfied for all kinds of defects and the composite laminated plate.

(3) Adhesive layer is not considered separately.

The model of carbon fiber reinforced composite materials with three-dimensional orthotropic is established and the mechanical parameters are showed in Tab. 1. Other physical parameters are concluded as following: density $\rho$ 
is $1340 \mathrm{~kg} / \mathrm{m}^{3}$, specific heat capacity $c$ is $700 \mathrm{~J} / \mathrm{kg} \cdot \mathrm{K}$, the heat transfer coefficient $\lambda_{1}$ is $12 \mathrm{~W} / \mathrm{m} \cdot \mathrm{K}$ along the fiber direction and the heat transfer coefficient $\lambda_{2}$ is $0.78 \mathrm{~W} / \mathrm{m} \cdot \mathrm{K}$ perpendicular to the fiber direction.

Tab. 1 Mechanical parameters of CFRP

\begin{tabular}{ccccccccc}
\hline \multicolumn{3}{c}{$\begin{array}{c}\text { Young's modulus } \\
(\mathrm{GPa})\end{array}$} & \multicolumn{3}{c}{$\begin{array}{c}\text { Shear modulus } \\
(\mathrm{GPa})\end{array}$} & \multicolumn{3}{c}{ Poisson's ration } \\
\hline$E_{1}$ & $E_{2}$ & $E_{3}$ & $G_{1}$ & $G_{2}$ & $G_{3}$ & $v_{12}$ & $v_{13}$ & $v_{23}$ \\
1.96 & 1.96 & 8.8 & 6.4 & 4.1 & 4.1 & 0.34 & 0.34 & 0.34 \\
\hline
\end{tabular}

The model containing the delamination, matrix cracking and fiber breakage near the impact points is built and the delamination is classified as joint and non-joint delamination damages as shown in Fig. 2 (a). The mesh is compartmentalized by the eight-node hexahedron unit grid (DC3D8 units) of linear heat transfer. The number of the simplified model units is about 3758 . The grid division result is shown in Fig. 2 (b).

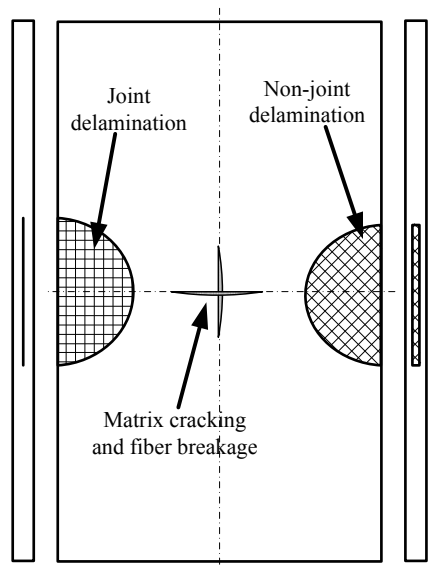

(a) Low-velocity impact damage

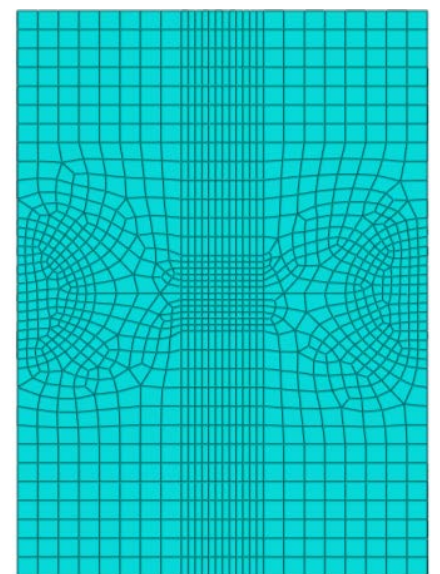

(b) The result of grid meshing
Fig. 2 Model for pulse stimulation and result of grid meshing

Suppose the environmental temperature $T_{e}$, with the value of $25^{\circ} \mathrm{C}$, which is unchangeable and equal to the initial temperature of the material.

The pulsed heat, with the heat flux density of 107 $\mathrm{W} / \mathrm{m}^{2}$, is selected for the thermal stimulation, and the pulsed heating duration is 2 milliseconds according to the current experimental equipment. The convective heat transfer coefficient $h_{c}$ of the material surface is 10 $W / m^{2} \cdot{ }^{\circ} \mathrm{C}$ in the cool-down process after heating. There is no internal heat source in the detection process and the radiation heat transfer on the surface is not considered in calculation because the growth of temperature is slight and the detection time is short.

The calculation process is divided into two steps according to the detection characteristics of pulsed thermal stimulation.

The first step is pulsed heating process:

Pulse heating duration:

The initial condition:

$$
t=0 \sim 2 \mathrm{~ms}
$$

$$
\left.T\right|_{t=0}=25^{\circ} \mathrm{C}
$$

The boundary conditions: $\left\{\begin{array}{l}-\left.\lambda \frac{\partial T}{\partial x}\right|_{x=0.004 \mathrm{~m}}=q=10^{7} \mathrm{~W} / \mathrm{m}^{2} \\ \left.\lambda \frac{\partial T}{\partial x}\right|_{x=0, x=100 \mathrm{~mm}}=\left.\lambda \frac{\partial T}{\partial y}\right|_{y=0, y=150 \mathrm{~mm}}=\left.\lambda \frac{\partial T}{\partial z}\right|_{z=4 m m}=h_{c}\left(T-T_{e}\right)\end{array}\right.$

where $T_{e}=25{ }^{\circ} \mathrm{C}, h_{c}=10 \mathrm{~W} / \mathrm{m}^{2} \cdot{ }^{\circ} \mathrm{C}$. The radiation heat transfer on the surface is not considered.

The second step is cooling process:

The cooling time:

$$
t=2 \mathrm{~ms} \sim 100 \mathrm{~s} \text {. }
$$

The calculated results of the first step are the initial conditions in this process. And the time step is $0.1 \mathrm{~s}$ in order to ensure the calculation accuracy.

The boundary conditions are the same as the first step except the pulsed heat flux:

$\left.\lambda \frac{\partial T}{\partial x}\right|_{x=0, x=100 \mathrm{~mm}}=\left.\lambda \frac{\partial T}{\partial y}\right|_{y=0, y=150 \mathrm{~mm}}=\left.\lambda \frac{\partial T}{\partial z}\right|_{z=4 m m}=h_{c}\left(T-T_{e}\right)$

3.2. Numerical simulation using ultrasonic infrared thermography

The material parameters of this model are the same with those in the above model. This model is gridded by the unit of the force heat coupling eight nodes hexahedron, C3D8RT (three-directional linear displacement, three-directional linear temperature, reduction integral and the hourglass control), and the face-to-face contact units are covered on the damage interface, simulating the collision and the friction on the surface of the contact. The total number of units is approximately 14000 , as shown in Fig. 3.

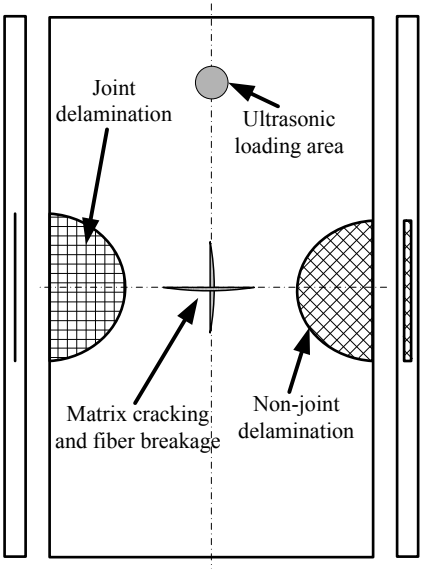

(a) Low-velocity impact damage

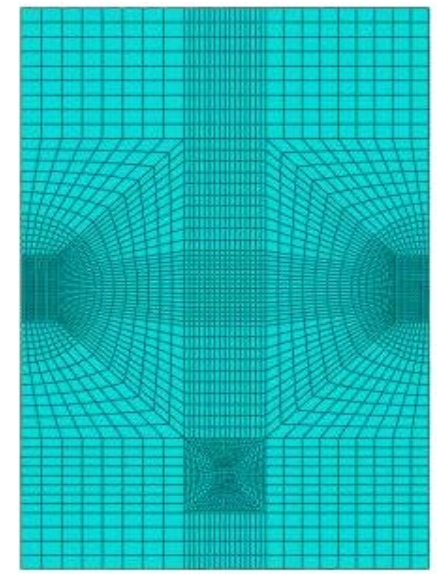

(b) The result of grid meshing

Fig. 3 Model for the ultrasonic stimulation and result of grid meshing

Suppose that the environmental temperature $T_{e}$, with the value of $25^{\circ} \mathrm{C}$, which is unchangeable and equal to the initial temperature of the material.

For convenience to calculation, the coefficient of static friction $\mu_{s}$, the coefficient of kinetic friction $\mu_{d}$ and coefficient of dynamic and static friction $\beta$ are $0.4,0.35$ and 5 respectively. The ultrasonic thermal stimulation is a harmonic displacement function $a(t)$, which is acted on the surface of the specimen inspected. In the entire process, the convective heat transfer coefficient $h_{c}$ of the material 
surface is $10 \mathrm{~W} /\left(\mathrm{m}^{2} \cdot \mathrm{K}\right)$. Because the temperature nearby the damage is low and the stimulation duration just lasts for a few hundred milliseconds, the radiation heat transfer nearby the damage area is ignored in the process of calculation.

According to the detection characteristic of ultrasonic thermal stimulation, the calculation contains two steps:

The first step is the ultrasonic stimulation process:

Stimulation duration:

$$
t=0 \sim 40 \mathrm{~ms}
$$

The initial condition:

$$
\left.T\right|_{t=0}=25{ }^{\circ} \mathrm{C}
$$

The boundary conditions:

(1) The two sides of the specimen are fixed (i.e., the six degrees of freedom were constrained).

(2) The harmonic displacement load is exerted on the front surface of the specimen.

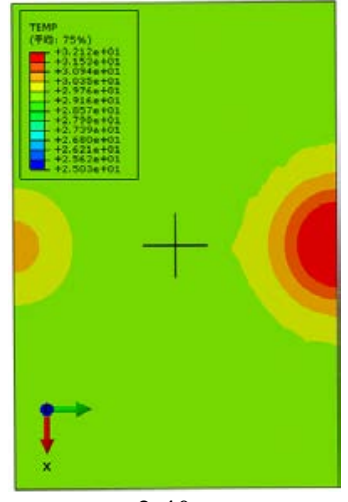

$2.40 \mathrm{~s}$

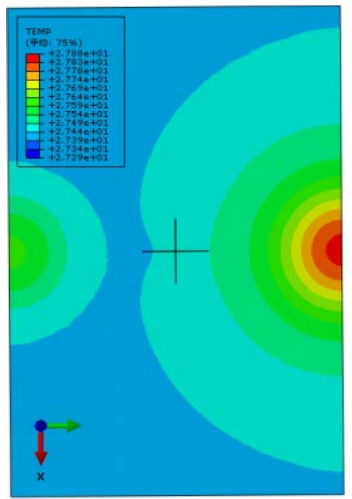

$22.05 \mathrm{~s}$

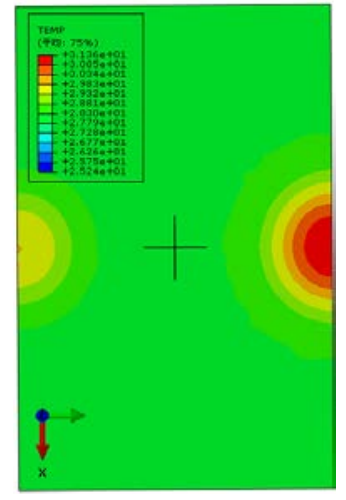

$4.50 \mathrm{~s}$

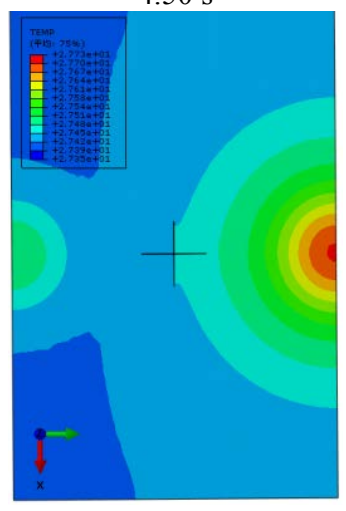

$24.60 \mathrm{~s}$

$a(t)=A \sin (2 \pi f t)$

Where, $f$ is the stimulation frequency with the value of 20 $\mathrm{kHz} . A$ is the stimulation amplitude with the value of 10 $\mu \mathrm{m}$.

The second step is the cooling process:

The cooling time:

$$
t=40 \sim 120 \mathrm{~ms}
$$

Calculation results of the first step are the initial conditions.

The boundary conditions are the same as the first step except for the harmonic displacement load.

\subsection{Results and analysis}

Fig. 4 shows the nephogram sequence of the surface at different time point by pulse infrared thermography.

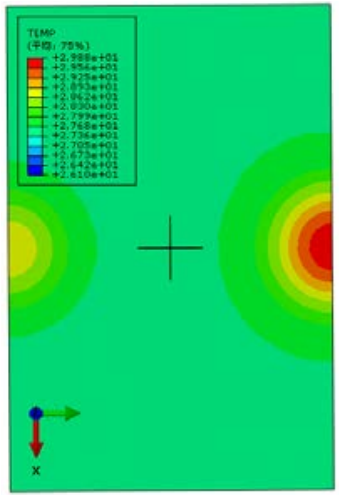

$9.00 \mathrm{~s}$

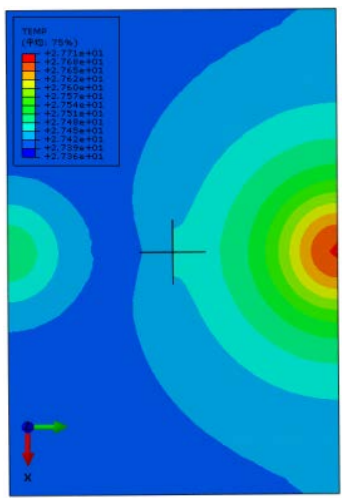

$25.05 \mathrm{~s}$

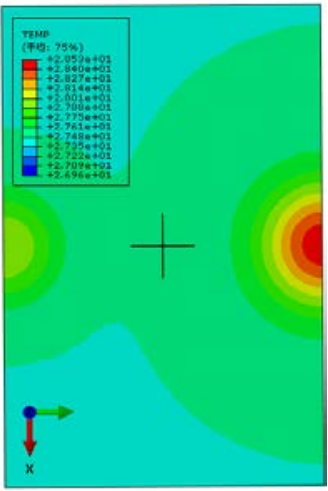

$15.30 \mathrm{~s}$

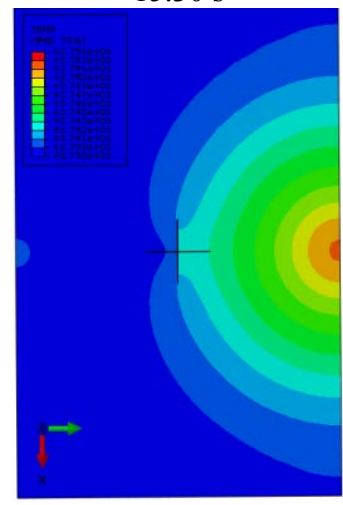

$30.00 \mathrm{~s}$

Fig. 4 Nephogram sequences of simulation results by pulse infrared thermography.

As the sequence diagrams display, it can be concluded that the pulse infrared thermography has significant effect on detecting delamination damage but no effect on matrix cracking and fiber breakage. For the delamination damage, the detect results of the non-joint delamination are better than the joint delamination. At 2.40s after heating, the contour of the non-joint delamination damage is greatly clear, while the corresponding surface of the joint damage is only a small area. This is because the two hierarchical interfaces of the joint delamination damage are relatively close, where exists only a few microns thickness of air, as a result the thermal resistance effect is insignificant and most of the heat can pass through this region. While there is a larger distance between the two layered interfaces for the non-joint delamination damage, where exists several millimeters of air. As a result, the thermal resistance effect is more significant and the heat does not pass through this region and accumulates in the upper layer interface, leading to the high temperature zone on the surface. In addition, it is also known that the pulse infrared thermography has no ability for the detection of matrix cracking and fiber breakage.

In the cooling process, the cooling at the high temperature side (non-joint delamination damage) is relatively slow owing to accumulating abundant heat, while the cooling at the low temperature (joint delamination damage) is fast because of the barriers of air within the matrix cracking for the thermal conductivity, which can be seen at 24.60 s and 25.05 s from the Fig. 4 . For the matrix cracking is usually along the fiber direction, the boundary line of high and low temperature along the layer direction is displayed in the thermal map. Generally, the 
boundary line between high and low temperature that can characterize the crack is produced in the thermal map when the temperature difference exists on both sides of crack.

Fig. 5 shows the nephogram sequence of the surface at different time point by ultrasonic infrared thermography. As described in the nephogram diagram, the matrix cracking and fiber breakage located in the center begin to appear at $1 \mathrm{~ms}$ after ultrasonic stimulation and gradually spreads to the surroundings. At $40 \mathrm{~ms}$, it has spread to become an approximate circular area. The heat conduction is hampered and accumulates due to the matrix cracking and fiber breakage at the center of the cross, resulting in the high-temperature zone in the nephogram. While the joint delamination damage located in the left begins to appear

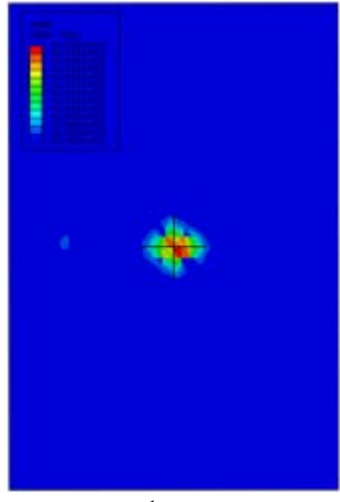

$1 \mathrm{~ms}$

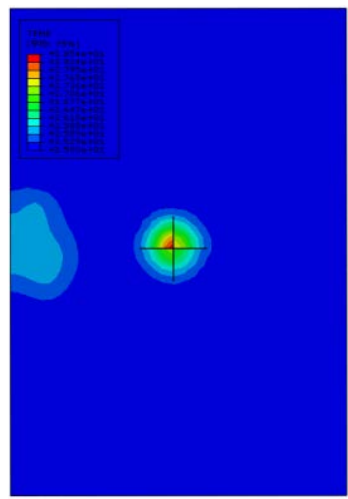

$40 \mathrm{~ms}$

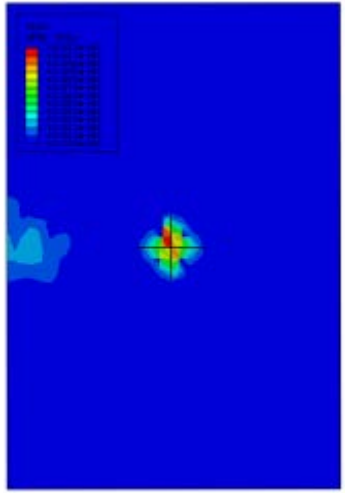

$3 \mathrm{~ms}$

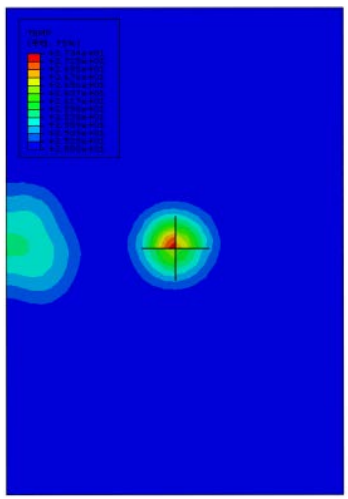

$60 \mathrm{~ms}$

and continuously extends to the surroundings until $3 \mathrm{~ms}$ due to the slow speed of the heat conduction along the thickness direction and the low thermal conductivity $(0.78)$ The surface temperature is keeping rising with the action of the stimulation. Eventually, the shape of the temperature field becomes a semicircle, which is consistent with that of the prefab delamination damage. The edge of the hot spot is vague due to the transverse thermal diffusion. In the entire excitation process, the temperature of the area containing matrix cracking and fiber breakage rises fast, and the temperature in the middle is greater than that in the tip. However, the surface temperature corresponding to the non-joint delamination does not change.

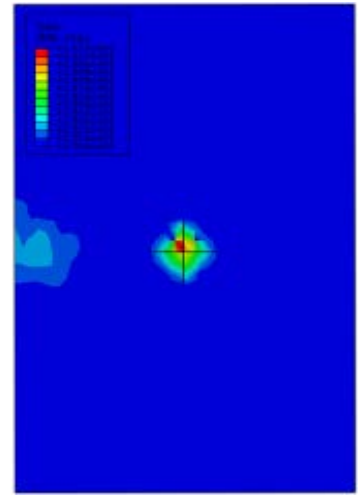

$5 \mathrm{~ms}$

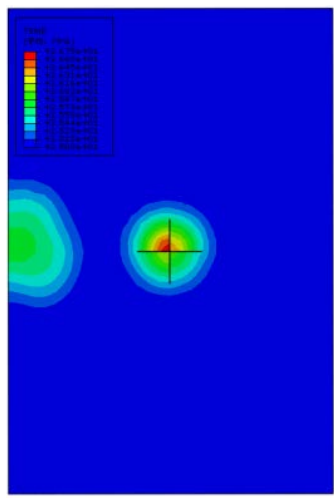

$80 \mathrm{~ms}$

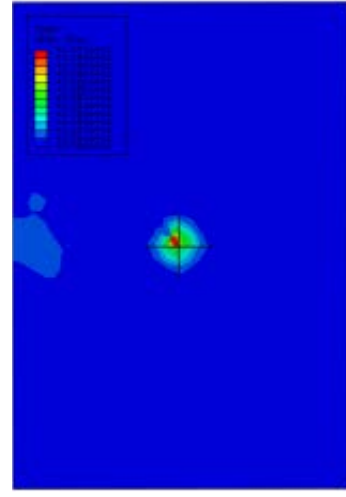

$20 \mathrm{~ms}$

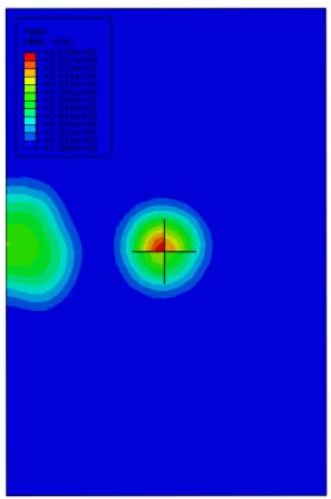

$100 \mathrm{~ms}$

Fig. 5 Nephogram sequences of simulation results by ultrasonic infrared thermography.

Fig. 6 displays the temperature-time curve at different points of the specimen under the ultrasonic stimulation. The rising section of the temperature-time at the matrix cracking and fiber breakage has the biggest slope, because the relative slip is smaller and the heat generated by friction is less. The energy generated in the middle of matrix cracking and fiber breakage is more than that at the tip, because the nodes in the middle possess relatively larger contact pressure and relative velocity. And the temperature curve also shows the phenomenon of fluctuation under ultrasonic stimulation, because the collision between the crack surfaces is intermittent and the temperature may drop during the intermittent period. The non-joint damage has no reaction under ultrasonic stimulation, because there is no effective contact interface at the damage and no heat produced by friction. The curve of the reference area at the bottom is almost a horizontal line, which indicates the temperature almost does not change.

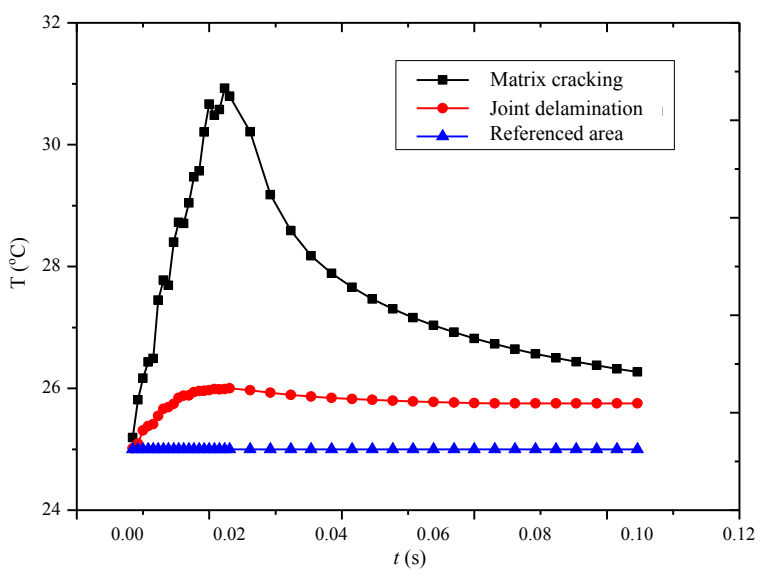

Fig. 6 Temperature-time curve of different location

Compared with the pulse infrared thermography, the ultrasonic infrared thermography does not contain pulse heating process and the temperature of the non-damage area also does not change, which is beneficial to determine 
and quantitatively analyze the defects or damage.

In summary, pulse infrared thermography is more sensitive to the damage with greater thermal resistance, such as non-joint delamination, while the ultrasonic infrared thermography is more sensitive to such damages as matrix cracking, joint delamination and fiber breakage. Combining the above two methods, the capability of the infrared thermography for detection will be greatly improved.

\section{Experimental investigation}

According to the results of numerical simulation, the pulse infrared thermography and ultrasonic infrared thermography have their own excellence in detecting low-velocity impact damage. Therefore, involving the combination of the two detection methods, the experiments on low-velocity impact damage detection are carried out in the following section.

\subsection{Materials and experimental equipment}

According to the last section, the specimen, made of carbon fiber reinforced composite laminated plates, is stacked in twelve layers with the size of $150 \mathrm{~mm} \times 100 \mathrm{~mm} \times 4 \mathrm{~mm}$ and extended in $\left[0^{\circ} / 45^{\circ} / 90^{\circ} /-45^{\circ}\right]_{12}$.

Six specimens, as shown in Fig. 7, with the serial number of $1 \# \sim 6 \#$ are chosen and successfully exposed to the low-velocity impact with the energy of $24.7 \mathrm{~J}, 26.8 \mathrm{~J}$, 23.7J, 28.7J, 31.6J, 36.7J respectively.

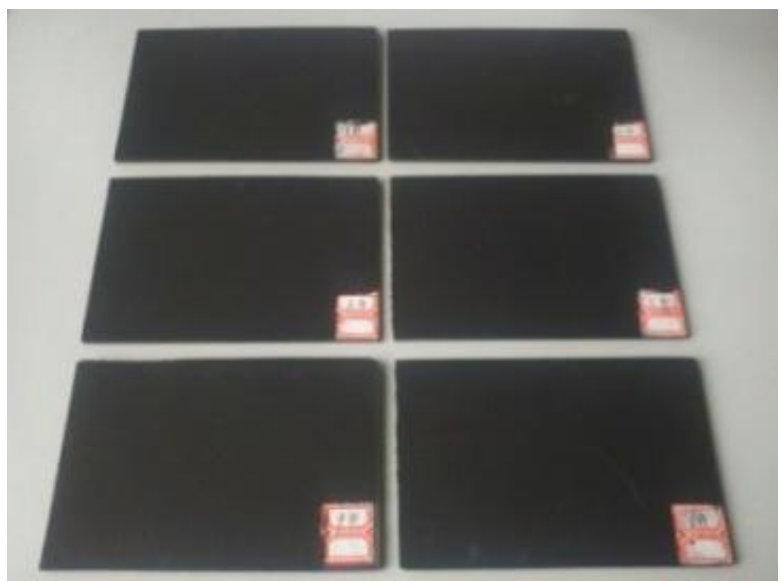

Fig. 7 Specimens of carbon fiber reinforced composites

Pulse infrared thermography detection system is mainly composed by the pulse stimulation device, infrared thermal imaging device, data acquisition, image processing and computer control system. The pulsed thermal stimulation device mainly utilizes two high energy xenon lamps to provide pulsed stimulation. The maximum energy of each lamp is $2.4 \mathrm{KJ}$ and the duration of thermal wave is $2 \mathrm{~ms}$. The lamps are installed in the mask to prevent the diffusion of light, so the relatively homogeneous light environment can be formed inside the cabinet.

The BRANSON pulse generator is introduced to be the ultrasonic stimulation, the maximum power of which is $4 \mathrm{~kW}$ with the stimulation frequency of $20 \mathrm{kHz}$. The output power can be adjusted by setting amplitude parameters and the load amplitude and ultrasonic stimulation duration of the ultrasonic gun can be adjusted by the controller.

The IR, produced by Infra Tec, with the type of Vhr680 can detect the temperature range of $-40^{\circ} \mathrm{C} \sim 1200^{\circ} \mathrm{C}$ with the sensitivity of $0.03^{\circ} \mathrm{C}$ at $30^{\circ} \mathrm{C}$, accuracy of $2 \%$ of reading, resolution of $640 \times 480$ pixels and data capture rate of 50 frames per second.

\subsection{Detection results by other non-destructive methods}

To distinctly observe the low-velocity impact damage in the specimen, scanning of electron microscope (SEM) and radiographic inspection technique (RT) are firstly applied to detect the impacted specimen. Taking 1\# specimens for instance, SEM and RT are applied for clearly observing the matrix cracking and fiber breakage on the surface/subsurface and delamination inside of the specimen, respectively. The partial results are shown in Fig. 8 and 9.

From the results of SEM, the matrix cracking and fiber breakage are clearly observed. The matrix cracking is along the direction of the fiber, while the fiber breakage is perpendicular to the direction of the fiber.

To obviously observe the delamination within the specimen, 6 detection sections, as shown in Fig. 9 are chosen along the direction of length with the $20 \mathrm{~mm}$ interval in the RT testing. From the results of RT, the delamination marked by the red loop in the sections (2)

(5) (i.e. in the middle of the specimen) can be observed. Moreover, the delamination caused by the low-velocity impact is more than one and distributed between different layers.

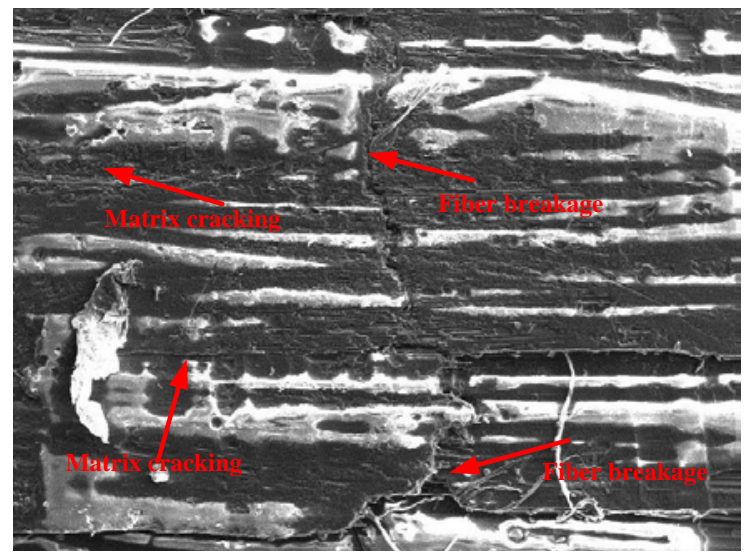

Fig. 8 The detection results using SEM 

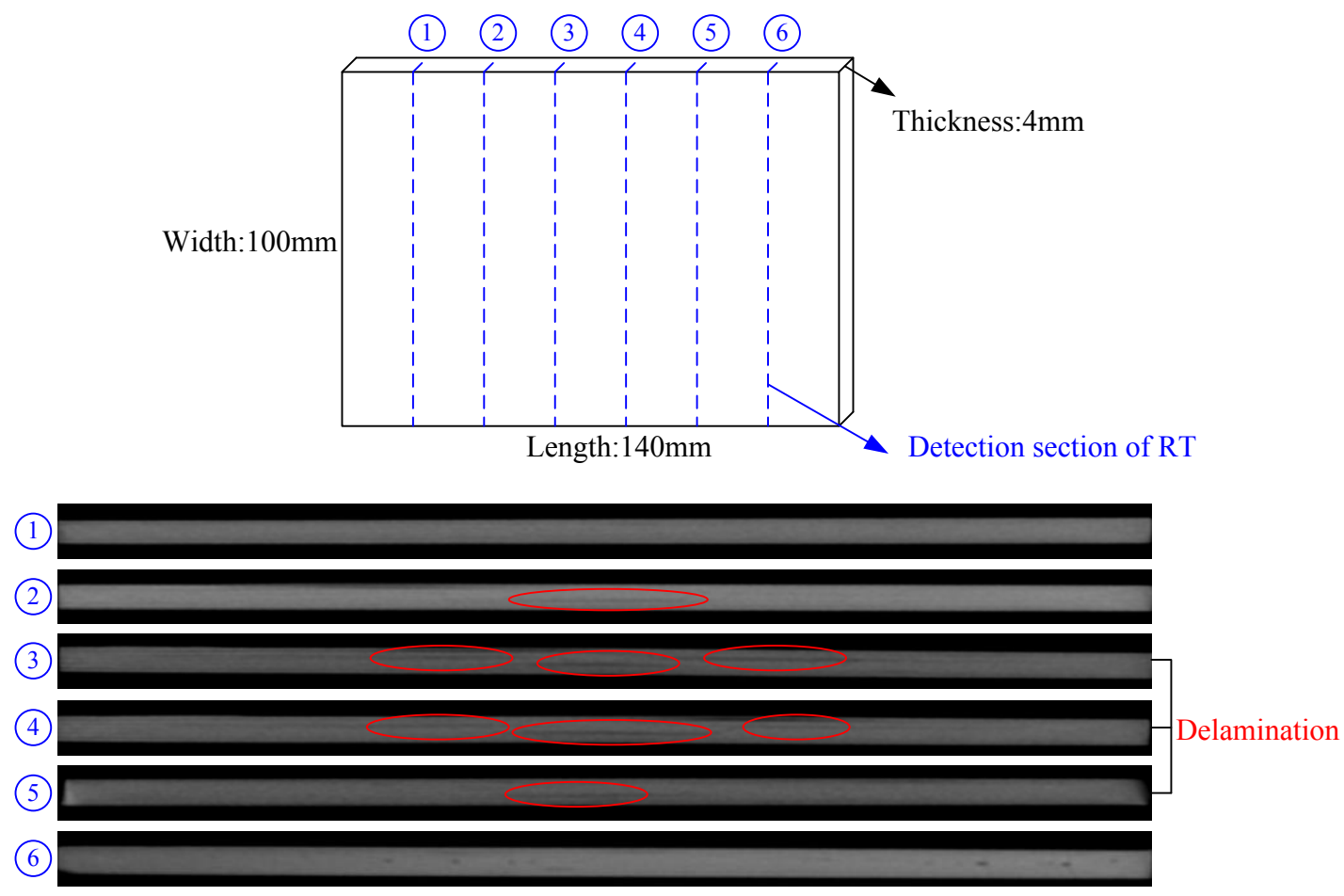

Fig. 9 The detection results using RT

\subsection{Hot spot extension in thermal map sequence}

Pulse infrared thermography and ultrasonic infrared thermography are applied to the specimens and the partial results are shown in Fig. 10 (a) and (b) respectively. In the detection test, we find the hot spot representing the damage gradually extend with the time after stimulation.

Taking 1\# specimen for instance, hot spot extension is analyzed. In pulse infrared thermography, the hot spot is along the direction of $-45^{\circ}$ at $0.20 \mathrm{~s}$ after stimulation and then extends to the direction of $90^{\circ}$ at $1.20 \mathrm{~s}$ in the middle of the specimen. From the thermal map sequence, we find a phenomenon that the hot spot gradually extends along the counter-clockwise direction and the lightness of the pre-emerging hot spot gradually fades. Why the phenomenon occurs is because the matrix breakage on the surface/subsurface immediately emerges when the specimen is exposed to the pulse stimulation and with transferring of the heat in the specimen the internal delamination damage also emerges. Therefore, this phenomenon (hot spot extension) not only indicates that there exists different damage types caused by low-velocity impact in the specimen but also qualitatively reveals the information that where the damage exists, which is consistent with the result of SEM and RT.

Similarly, hot spot extension can be observed in the detection by ultrasonic infrared thermography.

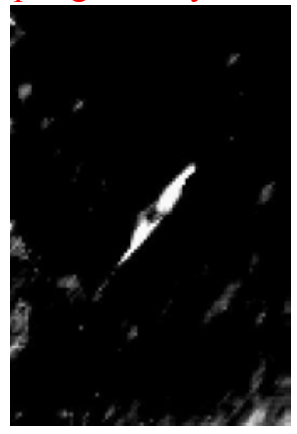

$0.20 \mathrm{~s}$

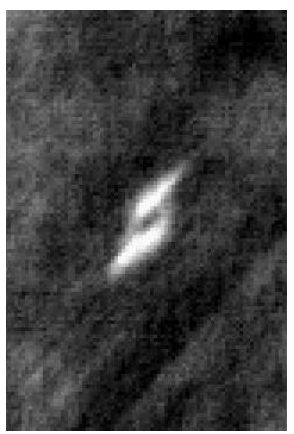

$2.10 \mathrm{~s}$

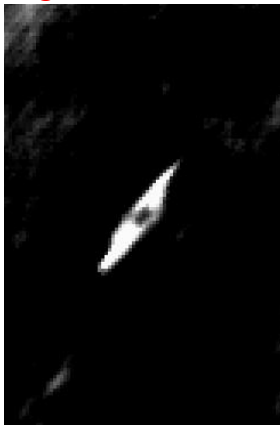

$0.40 \mathrm{~s}$

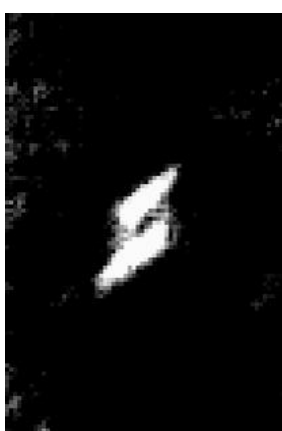

$4.10 \mathrm{~s}$

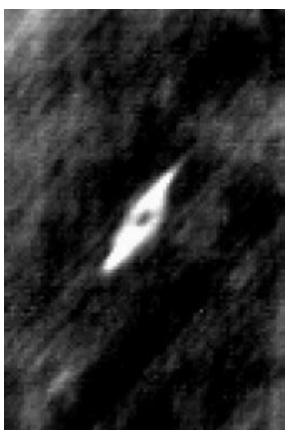

$1.20 \mathrm{~s}$

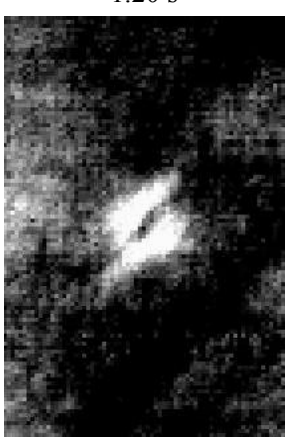

$7.60 \mathrm{~s}$

(a) Results by pulse infrared thermography 


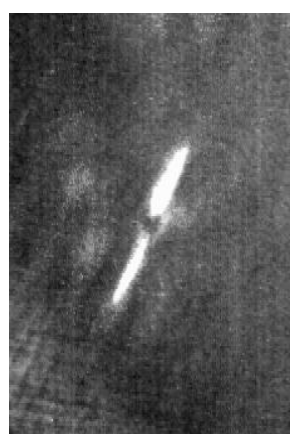

$0.08 \mathrm{~s}$

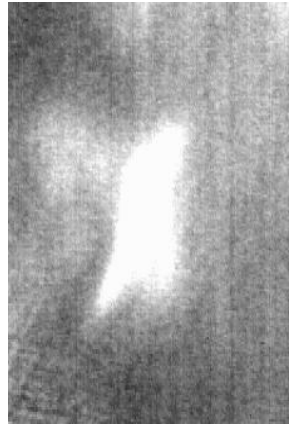

$0.92 \mathrm{~s}$

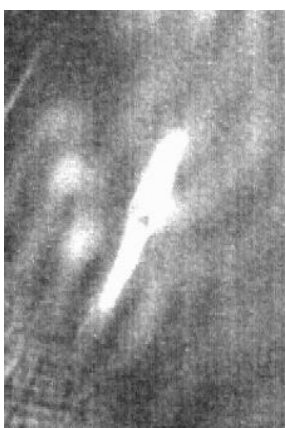

$0.16 \mathrm{~s}$

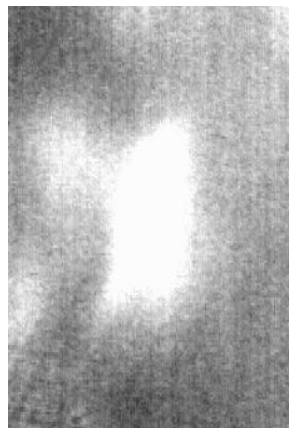

$1.92 \mathrm{~s}$

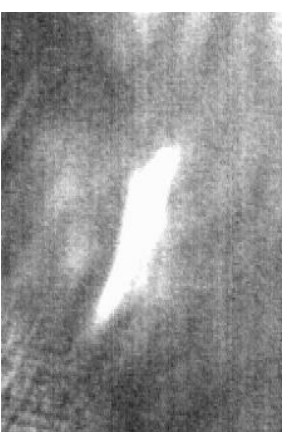

$0.56 \mathrm{~s}$

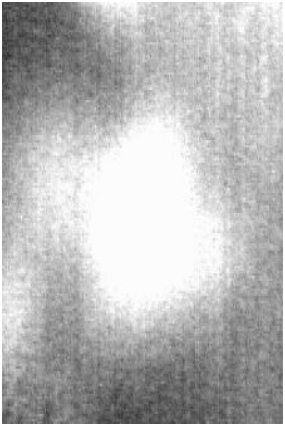

$4.90 \mathrm{~s}$

(b) Results by ultrasonic infrared thermography

Fig. 10 Partial detection results of 1 \# specimen

\subsection{Characterization for low-velocity impact damage}

\subsubsection{Characterization for delamination damage}

The morphology of delamination damage in partial specimens is showed in Fig. 11. Fig. 11 (a) and (b) show the thermal maps using pulse and ultrasonic infrared thermography respectively.

As is known from the Fig. 11, both the pulse infrared thermography and the ultrasonic infrared thermography have effective detection for delamination damage. Overall, the delamination damage is characterized as the relatively continuous block hot spot. There are dark areas in the hot spot center using pulse infrared thermography and the reasons mainly come from two parts. One is that the density at the impact point is slightly greater than that of the surrounding area and the heat transfer rate is slightly less than that of the surrounding area, which hinders heat transfer. The other is that there may be small range of matrix cracking, leading to the discontinuous of hot spot. The hot spot corresponding to that using the ultrasonic infrared thermography is more continuous and has the clear boundaries.

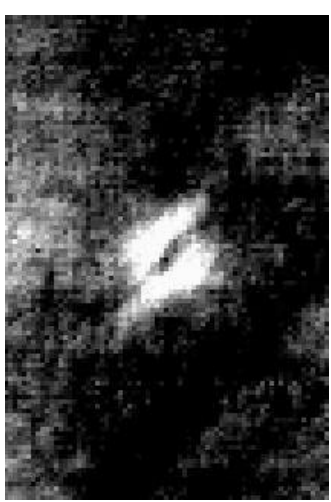

$1 \#$ front surface

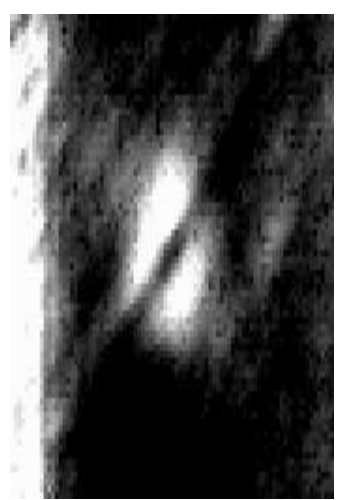

$4 \#$ front surface

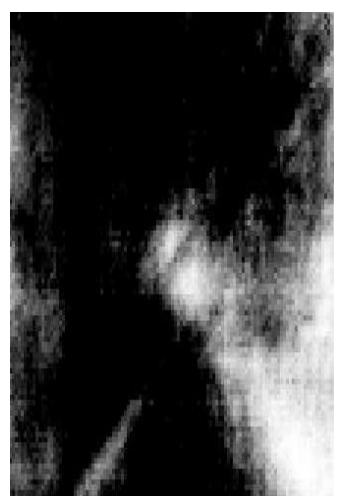

$5 \#$ front surface

(a) The results using pulse infrared thermography 


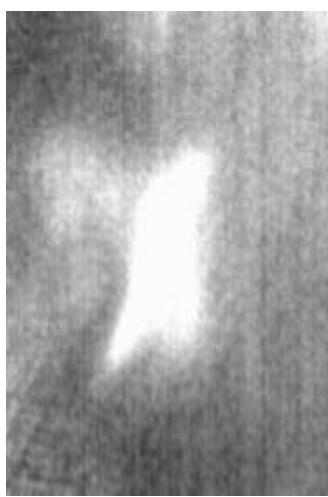

1\# front surface

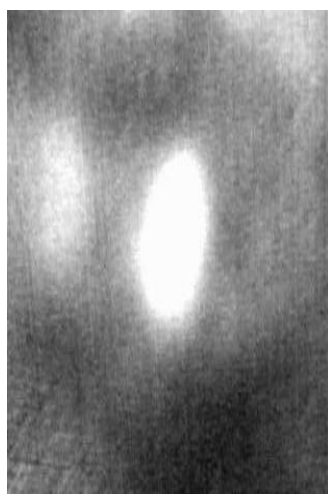

4\# front surface

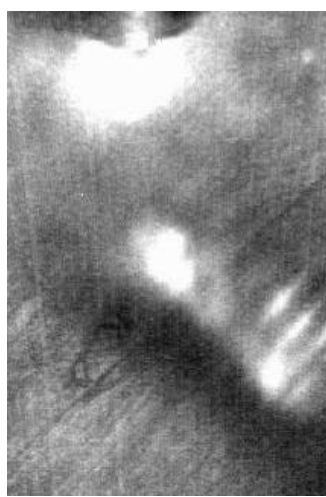

$5 \#$ front surface

(b) The results using ultrasonic infrared thermography

Fig. 11 Thermography of delamination using infrared thermography

\subsubsection{Characterization for the matrix cracking}

The morphology of the matrix cracking damage in partial specimens is displayed in Fig. 12. Fig.12 (a) and (b) represent the thermal maps using pulse and ultrasonic infrared thermography respectively.

There are different levels of matrix cracking damage on the $1 \# \sim 6 \#$ specimens after impact by infrared thermography. The scope and degrees of the damage on the back are greater than the impact on surface. Because the matrix cracking is along the fiber direction, when matrix cracking occurs, where the heat conduction is hindered to accumulate, it can be characterized as the hot spot with straight line shape, as shown in the Fig. 12.

Compared with pulse infrared thermography, ultrasonic infrared thermography can be applied to detect the surface matrix cracking obviously, as shown in Fig. 12 (b). Moreover, the subsurface matrix cracking (internal matrix cracking in the direction of the $45^{\circ}$ of the $3 \#$ specimen) can be also detected by ultrasonic infrared thermography.

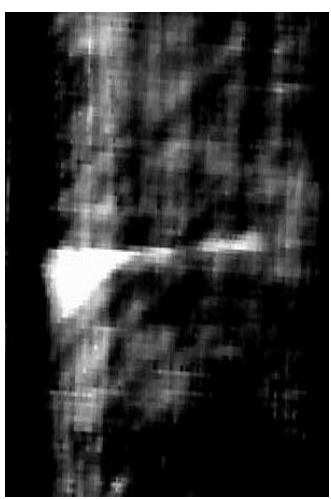

$3 \#(0.34 \mathrm{~s})$

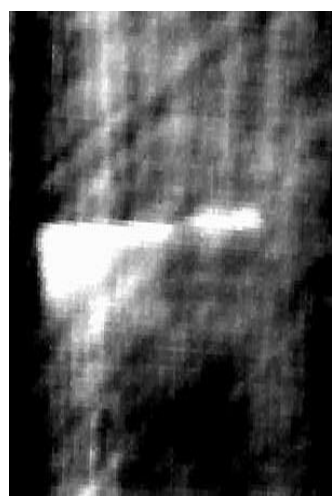

$3 \#(0.72 \mathrm{~s})$

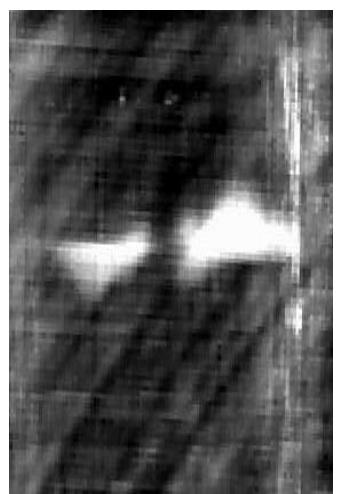

$4 \#(0.50 \mathrm{~s})$

(a) The results using pulse infrared thermography

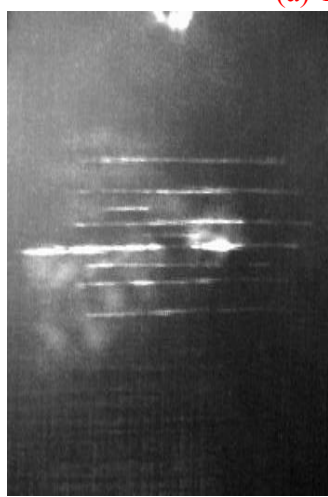

$3 \#(0.06 \mathrm{~s})$

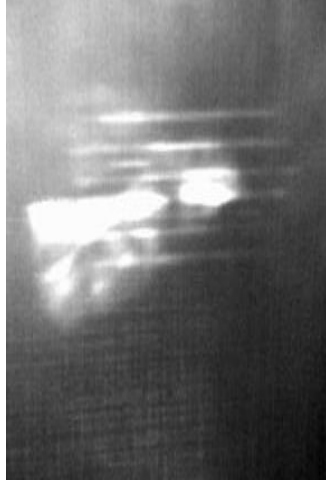

$3 \#(0.44 \mathrm{~s})$

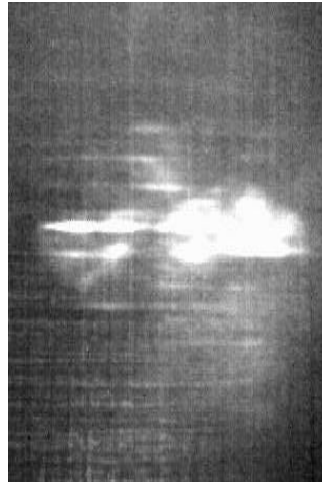

$4 \#(0.22 \mathrm{~s})$

(b) The results using ultrasonic infrared thermography

Fig. 12 Thermography of matrix cracking using infrared thermography

\subsubsection{Characterization for the fiber fracture}

With the increase of impact energy, the fiber breakage can be observed except for the pits with certain depth and matrix cracking on the surface of the specimen. Fiber breakages occur on the positive specimens of $4 \#, 6 \#$ and the opposite of $5 \#$ using infrared thermography, as shown in Fig. 13.

As is shown in Fig. 13, the fiber breakage of the specimen is characterized as "工" shape. Where the fiber 
breakage usually occurs is nearby the impact point. With the increase of impact energy, the possibility that fiber breakage occurs and fracture degree also increase gradually. Moreover, the degree of damage on the back of the specimen is often more extreme than that on the surface. According to the detection results, the pulse infrared thermography and the ultrasonic infrared thermography are capable to detect the fiber breakage that occurs on the surface of the specimen. But due to the short duration of the hot spots that represent the fiber fracture, the hot spots will fade away and be cover with the nearby hot spots those represent the matrix cracking and delamination damage.

In contrast, ultrasonic infrared thermography is more suitable for detecting the fiber breakage because the tiny contact surface is produced when the fiber breakage occurs, as a result, the friction heat will be easily generated after the ultrasonic stimulation and showed by the line-shape hot spots perpendicular to the direction of the fiber on the surface. Whereas, the capability for detecting the tiny joint damage is weak using the pulse infrared thermography and the fiber breakage damage is usually manifested by the block hot spots those characterize the small delamination area in the thermal map (as showed in Fig. 13 (a) 5\#), which, in this case, is easily mistaken for delamination damage located in the shallow surface layer.

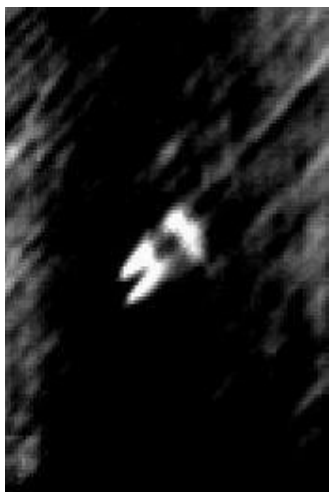

4\# (front surface)

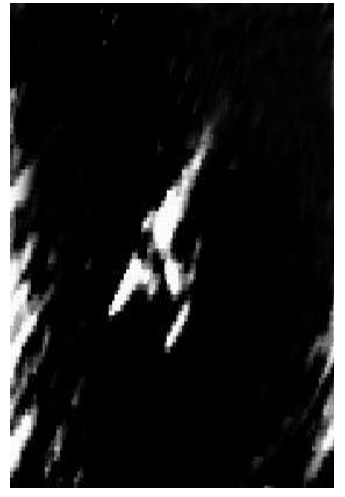

6\# (front surface)

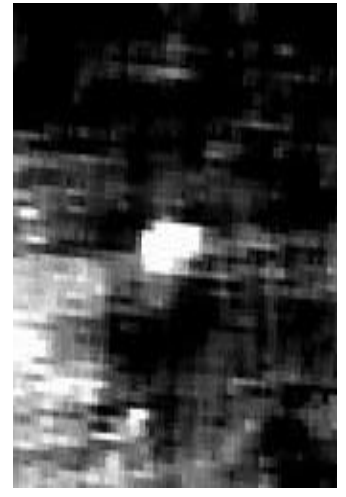

5\# (back surface)

(a) The results using pulse infrared thermography

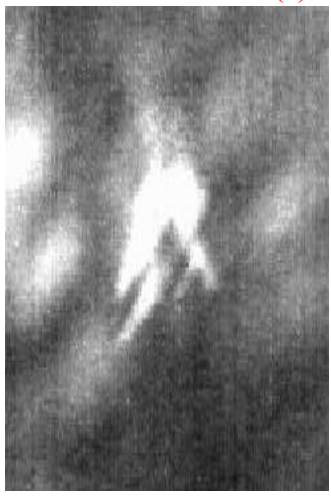

4\# (front surface)

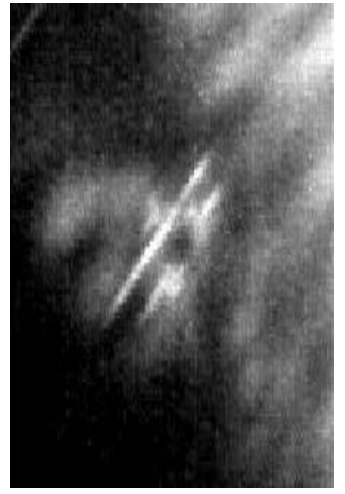

6\# (front surface)

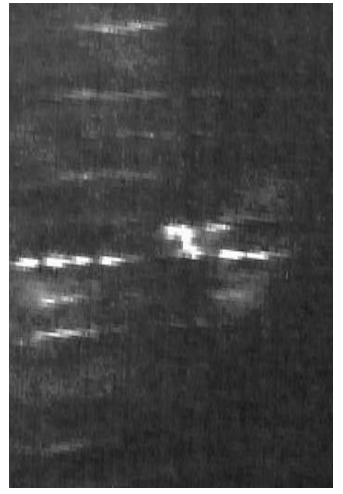

5\# (back surface)

(b) The results using ultrasonic infrared thermography

Fig. 13 Thermography of fiber fracture using infrared thermography

\section{Conclusion}

In this work, the investigation of impact damage detection in CFRP is carried out by numerical simulation and experiment. Numerical simulation is firstly analyzed to find that the pulse infrared thermography and ultrasonic infrared thermography have their own excellence in detecting low-velocity impact damage and the capability of the infrared thermography for detection will be greatly improved by combination of the two methods. Afterwards, the experimental investigation is carried out by the two methods. The following conclusions are obtained.

Pulse infrared thermography is more suitable for detecting the delamination damage, especially damage with large area and non-joint delamination, and ultrasonic infrared thermography is superior to detect the tiny damage, such as joint delamination, matrix cracking and fiber breakage. The combination of the above two methods can greatly improve the capability for detecting and evaluating the impact damage.

The delamination damage, matrix cracking and fiber breakage within the CFRP have their own specific characterizations in the thermal maps. The delamination damage, matrix cracking and fiber breakage are characterized as the block-shape hot spot, line-shape hot spot, and "工" shape hot spot respectively.

\section{Acknowledgements}

This work was supported by the Chinese National Science Foundation under Contract No. 51305447, No. 51575516.

\section{References}


[1] Naderi M, Kahirdeh A, Khonsari M M. Dissipated thermal energy and damage evolution of Glass/Epoxy using infrared thermography and acoustic emission [J]. Composites Part B: Engineering, 2012, 43(3): 1613-1620.

[2] Sfarra S, Ibarra-Castanedo C, Santulli C, et al. Falling weight impacted glass and basalt fiber woven composites inspected using non-destructive techniques [J]. Composites Part B: Engineering, 2013, 45(1): 601-608.

[3] Garnier C, Pastor M L, Eyma F, et al. The detection of aeronautical defects in situ on composite structures using Non Destructive Testing [J]. Composite structures, 2011, 93(5): 1328-1336.

[4] Castaings M, Singh D, Viot P. Sizing of impact damages in composite materials using ultrasonic guided waves [J]. NDT \& E International, 2012, 46: 22-31.

[5] Zou Y, Du D, Chang B, et al. Automatic weld defect detection method based on Kalman filtering for real-time radiographic inspection of spiral pipe [J]. NDT \& E International, 2015, 72: 1-9.

[6] Kuhn E, Valot E, Herve P. A comparison between thermosonics and thermography for delamination detection in polymer matrix laminates [J]. Composite Structures, 2012, 94(3): 1155-1164.

[7] Petrucci R, Santulli C, Puglia D, et al. Impact and post-impact damage characterization of hybrid composite laminates based on basalt fibers in combination with flax, hemp and glass fibers manufactured by vacuum infusion [J]. Composites Part B: Engineering, 2015, 69: 507-515.

[8] Maldague X, Largouët Y, Couturier J P. A study of defect depth using neural networks in pulsed phase thermography: modeling, noise, experiments [J]. Revue générale de thermique, 1998, 37(8): 704-717.

[9] Mian A, Han X, Islam S, et al. Fatigue damage detection in graphite/epoxy composites using sonic infrared imaging technique [J]. Composites Science and Technology, 2004, 64(5): 657-666.

[10] Lefèvre F, Le Niliot C. Multiple transient point heat sources identification in heat diffusion: application to experimental 2D problems [J]. International journal of heat and mass transfer, 2002, 45(9): 1951-1964.

[11] Louaayou M, Naït-Saïd N, Louai F Z. 2D finite element method study of the stimulation induction heating in synchronic thermography NDT [J]. NDT \& E International, 2008, 41(8): 577-581.

[12] Junyan L, Liqiang L, Yang W. Experimental study on active infrared thermography as a NDI tool for carbon-carbon composites [J]. Composites Part B: Engineering, 2013, 45(1): 138-147.

[13] Milne J M, Reynolds W N. The non-destructive evaluation of composites and other materials by thermal pulse video thermography [C]//1984 Cambridge Symposium. International Society for Optics and Photonics, 1985: 119-122.

[14] Tamma K K, Yurko A A. A unified finite element modeling/analysis approach for thermal-structural response in layered composites [J]. Computers \& structures, 1988, 29(5): 743-754.

[15] Savoia M, Reddy J N. Three-dimensional thermal analysis of laminated composite plates [J]. International Journal of Solids and Structures, 1995, 32(5): 593-608.

[16] Noack J, Rolfes R, Tessmer J. New layerwise theories and finite elements for efficient thermal analysis of hybrid structures [J]. Computers \& structures, 2003, 81(26): 2525-2538.

[17] Avdelidis N P, Almond D P, Dobbinson A, et al. Aircraft composites assessment by means of transient thermal NDT [J]. Progress in Aerospace Sciences, 2004, 40(3): 143-162.

[18] Ravikiran N K, Venkataramanaiah A, Bhat M R, et al. Detection and Evaluation of Impact damage in CFRP Laminates Using Ultrasound C-Scan and IR Thermography $[\mathrm{C}] /$ Proceedings of National Seminar NDE-06, Hyderabad. 2006.

[19] Usamentiaga R, Venegas P, Guerediaga J, et al. Automatic detection of impact damage in carbon fiber composites using active thermography [J]. Infrared Physics \& Technology, 2013, 58: 36-46.

[20] Genest M, Martinez M, Mrad N, et al. Pulsed thermography for non-destructive evaluation and damage growth monitoring of bonded repairs [J]. Composite Structures, 2009, 88(1): 112-120.

[21] Montanini R. Quantitative determination of subsurface defects in a reference specimen made of Plexiglas by means of lock-in and pulse phase infrared thermography $[\mathrm{J}]$. Infrared Physics \& Technology, 2010, 53(5): 363-371.

[22] Favro L D, Thomas R L, Han X, et al. Sonic infrared imaging of fatigue cracks [J]. International journal of fatigue, 2001, 23: 471-476.

[23] Maldague X P V. Introduction to NDT by active infrared thermography [J]. Materials Evaluation, 2002, 60(9): 1060-1073. 
[24] LIU Hui. Research on ultrasonic infrared lock-in thermography for non-destructive testing [D]. Harbin Institute of Technology, 2010. (In Chinese)

[25] SHEN Zhen, YANG Shengchun, CHEN Puhui. Experimental study on the behavior and characterization methods of composite laminates to withstand impact [J]. Acta Materiae Composite
Sinica, 2008, 25(5): 125-133. (In Chinese)

[26] Liu DB, Gao JJ, Guan ZD. A cumulative method for permanent indentation on laminates subjected to low-velocity impact $[\mathrm{C}] / 9^{\text {th }}$ China-Japan joint conference on composite materials. Huhhot, China, September 2010. 